\title{
The effect of superimposed high-cycle fatigue on thermo-mechanical fatigue in cast iron
}

Viktor Norman, Peter Skoglund, Daniel Leidermark and J ohan Moverare

The self-archived version of this journal article is available at Linköping University Electronic Press:

http:/ / urn.kb.se/ resolve?urn=urn:nbn:se:liu:diva- 121030

N.B.: When citing this work, cite the original publication.

Norman, V., Skoglund, P., Leidermark, D., Moverare, J ., (2016), The effect of superimposed high-cycle fatigue on thermo-mechanical fatigue in cast iron, International J ournal of Fatigue, 88, 121-131.

https:// dx.doi.org/ 10.1016/j.ijfatigue.2016.03.020

Original publication available at:

https:/ / dx.doi.org/ 10.1016/j.ijfatigue.2016.03.020

Copyright: Elsevier

http:// www.elsevier.com/

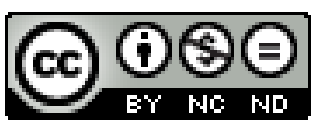




\title{
The effect of superimposed high-cycle fatigue on thermo-mechanical fatigue in cast iron
}

\author{
V. Norman ${ }^{\mathrm{a}, *}$, P. Skoglund ${ }^{\mathrm{a}, \mathrm{b}}$, D. Leidermark ${ }^{\mathrm{c}}$, J. Moverare $^{\mathrm{a}}$ \\ ${ }^{a}$ Division of Engineering Materials, Department of Management and Engineering, \\ Linköping University, SE-58183 Linköping, Sweden \\ ${ }^{b}$ Scania $C V$ AB, Materials Technology, SE-15187 Södertälje, Sweden \\ ${ }^{c}$ Division of Solid Mechanics, Department of Management and Engineering, Linköping \\ University, SE-58183 Linköping, Sweden
}

\begin{abstract}
The effect of superimposing a high-cycle fatigue strain load on an out-ofphase thermo-mechanical fatigue test of a lamellar, compacted and spheroidal graphite iron has been investigated. In particular, different total mechanical strain ranges, maximum temperatures and high-cycle fatigue strain ranges have been studied. From this, a new property has been identified, measured and compared, namely the thermo-mechanical and high-cycle fatigue threshold, defined as the high-cycle fatigue strain range at which the life is reduced to half. Using a previously developed model, the lifetimes and the threshold have been successfully estimated for the lamellar and compacted graphite iron, however underestimated for the spheroidal graphite iron. Nevertheless, an expression of the threshold was deduced based on the model, which possibly can estimate the value in other cast irons and its high-cycle fatigue frequency dependence.
\end{abstract}

Keywords: Cast iron, Thermo-mechanical fatigue, High-cycle fatigue, Fatigue crack growth, Life prediction

\section{Introduction}

Thermo-mechanical fatigue (TMF) has become a successively more important concept in the design of engine components in the heavy-vehicle automotive industry [1]. It refers to the fatigue damage accumulation due to

\footnotetext{
*Corresponding author. Phone: 004613284695

Email address: viktor.norman@liu.se (V. Norman) 
a combination of a conventional cyclic mechanical load, either displacement or load controlled, and a varying temperature [2,3]. Such a loading condition is typically encountered as the engine is exposed to repeated warming up and cooling down as it starts up and stops during every-day operation; a cycle often referred to as the start-operate-stop cycle [4].

The elevated material degradation is often observed in critical locations, in general in all parts where the thermal expansion of heated material is impeded by less hot surrounding material. This has been identified as one of the main limitations of the overall service lifetime of many engine components, as well as an obstacle in fulfilling the demands of higher efficiency and reduced exhaust emission. On top of this, there is an additional factor which has become an increasingly more studied research topic, namely the possible detrimental synergy effect of combined high-frequent mechanical loading, often referred to as superimposed high-cycle fatigue (HCF) [5-8]. Many materials, including aluminium, steels, superalloys and cast irons, have been proven to be sensitive to a superimposed HCF load and it is therefore important to characterise this effect [5-10]. In a previous study [10], it was identified that there exists a HCF load threshold independent of the underlying TMF cycle, beneath which the overall fatigue life is unaffected, in the compacted graphite iron EN-GJV-400.

The concerned components constituting a heavy-vehicle engine, such as the cylinder head, the cylinder block and the exhaust manifolds, are cast using different varieties of cast irons. The different grades of cast irons may differ significantly in mechanical properties but have a characteristic phase structure in common, consisting of graphite phase particles embedded in a steel matrix phase. The wide diversity in properties is often ascribed to the large variety of feasible graphite shapes and matrix structures, i.e. ferritic, pearlitic structures etc. These two parameters are governed by the phase transformations that occur during the cooling and are partly controlled by variation of the chemical melt composition, cooling conditions and inoculation.

To address the above mentioned problems a substantial effort has been put on the development of simulation-based design tools, employing finite element software and lifetime assessment modelling, in order to estimate the properties of new component designs as a more rapid substitute compared to prototype testing $[1,4,8,11]$. Such an approach should be of use for engine constructors to avoid employing inappropriate geometries from a TMF perspective. However, much research is still required for successful 
lifetime assessment models.

The purpose of this study is to present and compare the effect of combining superimposed high-cycle and thermo-mechanical fatigue on three representatives of different cast iron families, namely a lamellar (LGI), a compacted (CGI) and a spheroidal graphite iron (SGI). Presently, the existing TMF-HCF data of these materials are highly limited and no comprehensive comparison of different cast irons has yet been carried out regarding the TMF-HCF interaction. In particular, this study will report the HCF threshold levels and also employ the lifetime assessment model developed in [10].

\subsection{Thermo-mechanical fatigue and superimposed high-cycle fatigue}

A thermo-mechanical fatigue test is a special version of a low-cycle fatigue (LCF) test where the temperature is allowed to vary together with the mechanical load, which most commonly is strain controlled [2, 3]. The cycle period is normally the same for the thermal and mechanical cycle, however different phase shifts between the two are often studied. The two most commonly employed are the extremes cases, namely when the phase shift is zero, called in-phase testing, or $180^{\circ}$, also called out-of-phase testing. In the latter, the maximum temperature coincides with the minimum strain, while the opposite pertains for the former case.

If a HCF load is to be added, then the mechanical load will oscillate with some amplitude about the base signal which corresponds to the signal applied in the standard TMF test, see Fig. 1. As often done for straincontrolled tests $[7,10]$, the base strain signal is referred to as the thermomechanical fatigue strain $\varepsilon_{T M F}$ while the oscillating strain signal is denoted high-cycle fatigue strain $\varepsilon_{H C F}$. Added together, they will constitute the total mechanical strain $\varepsilon_{\text {Mech }}$ corresponding to the combined strain signal in Fig. 1b. The prefix total is included to emphasize that the variable is composed of two mechanical strain signals and pertains to a TMF-HCF condition. For consistency, the mechanical strain will be referred as the TMF strain when in a TMF condition for which the HCF strain is zero. Thus, in a TMF-HCF test, the uniaxial strain $\varepsilon$ measured by an extensometer is at any instant $t$ equal to

$$
\varepsilon(t)=\varepsilon_{T h}(t)+\varepsilon_{M e c h}(t)=\varepsilon_{T h}(t)+\varepsilon_{T M F}(t)+\varepsilon_{H C F}(t)
$$

where $\varepsilon_{T h}$ is the thermal strain representing the thermal expansion. The definitions of the different strain ranges, $\Delta \varepsilon_{M e c h}, \Delta \varepsilon_{T M F}$ and $\Delta \varepsilon_{H C F}$, follow accordingly, see Fig. 1. It is important to realise that the resulting 

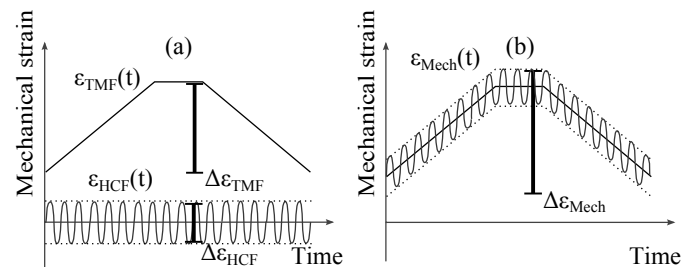

Figure 1: A schematic illustration of the TMF and HCF strain signals.

uniaxial engineering stress $\sigma(t)$ is only explicitly dependent on the applied mechanical strain and not on the induced thermal strain.

Only a handful of experimental studies have been carried out on the TMF-HCF behaviour of cast irons [6-8, 10]. Nevertheless, it has been reported for different cast irons that there exists a HCF strain range threshold $\Delta \varepsilon_{H C F}^{T h}$, henceforth referred to as the TMF-HCF threshold, below which the fatigue life is unaffected by the superimposed HCF load [6, 10]. However, the data is scarce and there are some inconsistencies between the different investigations. For instance regarding SGI, in which no threshold was observed by Beck [7] in opposition to the observations of Metzger et al. [8].

\subsection{Fatigue crack behaviour in cast iron under uniaxial loading}

Many investigations of the microscopic damage in monotonic and cyclic tension have been conducted on cast irons at room temperature [12, 13]. For instance, it has in monotonic tension been observed that the graphite and the steel matrix debond perpendicularly to the load direction at low levels of strain, namely about $0.03 \%$ for both LGI and SGI [13]. In addition, at the same level of straining, internal fracture of the graphite has been seen in the case of LGI, CGI and SGI [12]. Thus, the graphite phase appears to fracture at a very early stage, either within the phase itself or along the phase interface shared with the steel matrix. As the load is further increased, these early fracture events result in the development of small cracks, or microcracks, extending into the matrix at multiple locations [12]. At even higher load levels, the microcracks successively propagate and eventually coalescence.

Regarding cyclic loading, these microscopic fracture processes appear to be similar to the static case, namely the initiation, propagation and coalescence of microcracks, which are schematically illustrated in Fig. 2. Small fatigue cracks have been observed to emanate from graphite particles in both isothermal and thermo-mechanical fatigue [8, 14, 15]. More importantly, small crack initiation has also been concluded to occur within the 


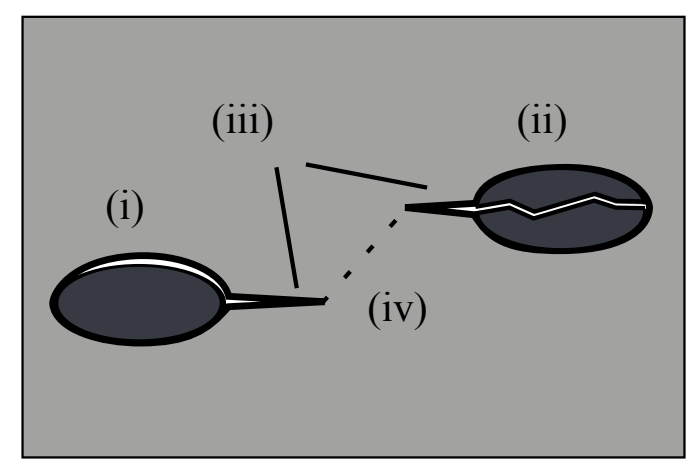

Figure 2: Schematic illustration of the microscopic fracture processes: (i) graphite-matrix debonding, (ii) internal graphite fracture, (iii) microcrack propagation into the matrix and (iv) microcrack coalescence.

first fatigue cycles of both the mentioned fatigue modes [14, 16], which is a commonly stated assumption in many life assessment approaches, e.g. $[8,10,16,17]$. The microcracks have been experimentally verified to initiate at multiple graphite locations for LGI, CGI and SGI [14, 15], and the following propagation regime is associated with individual microcrack propagation and subsequent onset of microcrack coalescence [8, 14, 15]. In fact, Socie and Fash [14] observed that the microcrack length at the moment before the final coalescence resulting in the final fracture plane, was about $1-2 \mathrm{~mm}$ in all three cast iron types, LGI, CGI and SGI. Nonetheless, it should also be noted that the distinction between fatigue crack initiation and propagation might be somewhat unclear because of the discontinuous growth of microcracks into a crack of macroscopic proportions.

Casting defects and the free surface might also have a certain impact on fatigue crack behaviour [17, 18], in particular in the case of high temperature [16]. Defects have been proven to have a significant influence on the fatigue properties of SGI, as fatigue crack initiation has been traced back to naturally occurring casting defects such as microshrinkages [18]. Furthermore, in an earlier study of Nadot et al. [17] it was established that the crack propagation rate of microcracks emanating from casting defects at the surface was similar to the long crack propagation rate when the crack length was above a certain small crack transition size of $120 \mu \mathrm{m}$. Oxidation due to an ambient environment has also been shown to be of importance, both at room temperature [17] and at elevated temperatures [19].

Some of the investigations on TMF and TMF-HCF testing of cast irons have also studied the associated crack behaviour $[5,7,8]$, however presently 
only on SGI material. Hammers et al. [5] categorised different fatigue cracks into three different types depending on the type of crack, namely surface initiated, bridging two neighbouring nodules or nodule initiated, but did not mention the role of casting defects. However, it was concluded that the crack growth characteristic was unchanged when applying a superimposed $\mathrm{HCF}$ strain range, i.e. the crack length profile as a function of number of cycles normalised by the number of cycles to failure appeared to be the same regardless of the value of the HCF strain range.

\section{Lifetime assessment model}

The theoretical framework on fatigue life assessment employed in this work is based on the same approach that has been presented previously [10] and is only briefly reproduced here.

Firstly, as it is commonly assumed [8], it is suggested that the crack extension due to the TMF cycle and the HCF cycles, is separable,

$$
\Delta a=\Delta a_{T M F}+\sum_{i} \Delta a_{H C F}^{i}
$$

where $\Delta a_{T M F}$ is the crack extension due to one TMF cycle alone and $\Delta a_{H C F}^{i}$ are the crack extensions due to each HCF cycle $i$ elapsed during the TMF cycle.

Secondly, to address the observed fatigue damage process in which multiple microcracks are initiated and eventually coalesced, it is hypothesised that the average microcrack length follows a Paris law expression,

$$
\frac{d \bar{a}}{d N}=C\left[\Delta K_{I}(\Delta \sigma, \bar{a})\right]^{n}=C[\bar{Y} \Delta \sigma \sqrt{\pi \bar{a}}]^{n}
$$

where $\bar{a}$ is the average microcrack length, $N$ is the number of cycles, $\Delta K_{I}$ is the stress-intensity factor range in mode I loading of the average microcrack for a given stress range $\Delta \sigma, \bar{Y}$ is a constant related to the average crack geometry, $C$ and $n$ are the Paris law coefficient and exponent respectively. Dealing with average values, it is supposed that the initial crack size is comparable with the average graphite particle size, and the final crack length is the average microcrack length at the point of failure. It is further assumed that crack closure is negligible since the cracks are small, which is supported by studies of small cracks in many ductile materials [20] and defect-initiated microcracks in SGI [21]. Thus $\Delta \sigma$ is replaced with $\sigma_{\text {Max }}$ and the first term on the right-hand side of Eq. 2 can be written as 


$$
\Delta \bar{a}_{T M F}=C\left[\sigma_{M a x} \cdot \bar{Y} \sqrt{\pi \bar{a}}\right]^{n} \Delta N
$$

Thirdly, regarding the superimposed HCF load, it is assumed that only HCF cycles applied at a tensile stress level contribute to the crack propagation, since the cracks are expected to be closed at compressive stresses. This has been show to be a valid assumption for EN-GJV-400 by performing TMF-HCF tests with HCF cycles only at compressive stresses [10]. In addition, the temperature dependence of the Paris parameters is assumed to be negligible, hence $C$ and $n$ are seen as mean values applicable to both TMF and HCF cycles. It is important to note that this is an engineering assumption, which could be inaccurate. However, it is motivated since it adds simplicity and yields good life estimates which will be seen later. Accordingly, the HCF contribution to Eq. 2 is written as

$$
\sum_{i} \Delta \bar{a}_{H C F}^{i}=\rho C\left[\Delta \sigma_{H C F} \cdot \bar{Y} \sqrt{\pi \bar{a}}\right]^{n} \Delta N
$$

where $\rho$ is the average number of HCF cycles above the zero stress level during one TMF cycle. Combining Eqs. 2, 4 and 5, as well as putting $\Delta \sigma_{H C F}$ equal to $E \Delta \varepsilon_{H C F}$, integration yields

$$
D \int_{0}^{N_{f}}\left[\sigma_{M a x}(N)\right]^{n} d N=1-D \rho\left[E \Delta \varepsilon_{H C F}\right]^{n} N_{f}
$$

where $\sigma_{M a x}(N)$ is the maximum stress at every cycle obtained from a TMF test without HCF loading, $E$ is the elastic modulus, $N_{f}$ is the number of cycles to failure and $D$ is a constant for a given temperature cycle, equal to

$$
D=\frac{C(\bar{Y} \sqrt{\pi})^{n}}{\int_{\bar{a}_{0}}^{\bar{a} f} \bar{a}^{-n / 2} d \bar{a}}
$$

The numerical value of $\rho$ is calculated as the number of HCF cycles above zero stress in a stabilised hysteresis loop. The numerical values of $D$ and $n$ are obtained by fitting Eq. 6 to regular TMF tests, hence by setting the right-hand side to unity. The numerical values of $D, n$ and $\rho$ for 100$400^{\circ} \mathrm{C}$ and $100-500^{\circ} \mathrm{C}$ are presented in Table 1. It should also be noted that it is not possible to obtain values for $\bar{Y}$ and $C$ explicitly through the above approach since they are integrated into one inseparable constant, Eq. 7.

In order to reach Eq. 6 , the HCF strain range $\Delta \varepsilon_{H C F}$ was related to the HCF stress range $\Delta \sigma_{H C F}$ through an elastic modulus independent of the 
temperature. Thus, as similar to the treatment of the Paris parameters, the variation in the elastic modulus due to the temperature is disregarded and the value of the elastic modulus is approximated with the elastic modulus at $100^{\circ} \mathrm{C}$, given in Table 2 . This is considered a justified approximation since the cracks are closed during the hot part of the TMF cycle and since the variation in the elastic modulus in the concerned temperature range is considered insignificant, as seen for EN-GJV-400 [10].

Table 1: The numerical values of the fitted model parameters and estimated initial and final crack lengths.

\begin{tabular}{cccccc}
\hline Material & $n[-]$ & $D\left[M P a^{-n}\right]$ & $\rho[-]$ & $\bar{a}_{0}[\mu m]$ & $\bar{a}_{f}[\mu m]$ \\
\hline EN-GJL-250 & & & & & \\
$100-400^{\circ} \mathrm{C}$ & 9.89 & $1.33 \cdot 10^{-25}$ & 3311 & & \\
$100-500^{\circ} \mathrm{C}$ & 9.91 & $3.08 \cdot 10^{-25}$ & 3641 & 104.0 & 1000 \\
\hline EN-GJV-400 & & & & & \\
$100-400^{\circ} \mathrm{C}$ & 11.91 & $2.57 \cdot 10^{-33}$ & 3716 & & \\
$100-500^{\circ} \mathrm{C}$ & 12.65 & $9.95 \cdot 10^{-35}$ & 4243 & 74.3 & 1000 \\
\hline EN-GJS-SiMo5-1 & & & & & \\
$100-400^{\circ} \mathrm{C}$ & 8.47 & $1.44 \cdot 10^{-25}$ & 3364 & & \\
$100-500^{\circ} \mathrm{C}$ & 10.11 & $3.00 \cdot 10^{-29}$ & 4050 & 63.0 & 1000 \\
\hline
\end{tabular}

Table 2: Static mechanical properties at $25^{\circ} \mathrm{C}$ and elastic modulus at $100^{\circ} \mathrm{C}$ of EN-GJL250, EN-GJV-400 and EN-GJS-SiMo5-1.

\begin{tabular}{cccc}
\hline Material & $R_{p 0.2}[M P a]$ & $R_{m}[M P a]$ & $E[G P a]$ \\
\hline EN-GJL-250 & - & $263 \pm 13$ & $94 \pm<1$ \\
EN-GJV-400 & $311 \pm 10$ & $416 \pm 12$ & $154 \pm 2$ \\
EN-GJS-SiMo5-1 & $494 \pm<1$ & $587 \pm 11$ & $168 \pm<1$ \\
\hline
\end{tabular}

\subsection{TMF-HCF threshold expression}

From Eq. 6, an elegant expression of the TMF-HCF threshold $\Delta \varepsilon_{H C F}^{T h}$ can be obtained. Starting with the assumption that the maximum stress value is fairly constant, which is not unreasonable as it usually stabilises after a couple of cycles, the number of cycles to failure of a TMF-HCF test normalised to its corresponding value obtained in a TMF test is

$$
\frac{N_{f}\left(\Delta \varepsilon_{H C F}\right)}{N_{f}\left(\Delta \varepsilon_{H C F}=0\right)}=\frac{1}{1+\rho\left(\frac{E \Delta \varepsilon_{H C F}}{\sigma_{M a x}}\right)^{n}}
$$


where $\sigma_{\text {Max }}$ now denotes a constant maximum stress value. Defining the threshold as the $\Delta \varepsilon_{H C F}$ where the TMF-HCF life normalised with reference to the TMF life has fallen to a half, Eq. 8 can be rearranged as

$$
\Delta \varepsilon_{H C F}^{T h}=\frac{\sigma_{M a x}}{E}\left(\frac{1}{\rho}\right)^{\frac{1}{n}} \approx \frac{\sigma_{M a x}}{E}\left(\frac{2 f_{T M F}}{f_{H C F}}\right)^{\frac{1}{n}}
$$

where the number of HCF cycles above zero stress $\rho$ has been estimated as the ratio of the HCF and TMF cycle frequency, $f_{H C F}$ and $f_{T M F}$ respectively, which is equal to the total number of HCF cycles per TMF cycle, divided by two. Thus, it is assumed that the hysteresis loop is symmetric about the zero stress line, which however, is not entirely true for cast irons. On the other hand, the effect of tension-compression asymmetry is considered small in the context and is therefore neglected.

\subsection{Crack length profile}

As a consequence of the given lifetime assessment model, the average crack length as a function of number of cycles $\bar{a}(N)$ can be obtained. In the case of a regular TMF test, the crack length profile is acquired by integration of Eq. 3 and the substitution of $C(\bar{Y} \sqrt{\pi})^{n}$ using Eq. 7, which yields

$$
[\bar{a}(N)]^{1-\frac{n}{2}}=\bar{a}_{0}^{1-\frac{n}{2}}+D\left(\bar{a}_{f}^{1-\frac{n}{2}}-\bar{a}_{0}^{1-\frac{n}{2}}\right) \int_{0}^{N}\left[\sigma_{M a x}(N)\right]^{n} d N
$$

where $\bar{a}_{0}$ and $\bar{a}_{f}$ are the average initial and final crack length.

The average initial crack length is assumed to be in the same order as the average graphite particle size in the studied LGI and CGI, and in the order of the average microshrinkage diameter in SGI. For the two former, the average initial crack length is taken as the average projected graphite flake length, $\bar{l}_{\perp}$, in the plane perpendicular to the loading direction, since this is the plane in which microcracks most commonly grow. To obtain this value, it is assumed that the flake orientation is random and has a uniform probability density function $\psi$. Accordingly, the unit sphere can be seen as a continuous probability space in which an arbitrary flake orientation is represented by a set of spherical coordinates $(r=1, \varphi, \theta)$, see Fig. 3. Integration over the whole unit sphere should include all possible orientations, i.e. the accumulated probability must equal unity,

$$
\int_{0}^{2 \pi} \int_{0}^{\pi} \psi \sin \theta d \theta d \varphi=4 \pi f=1
$$




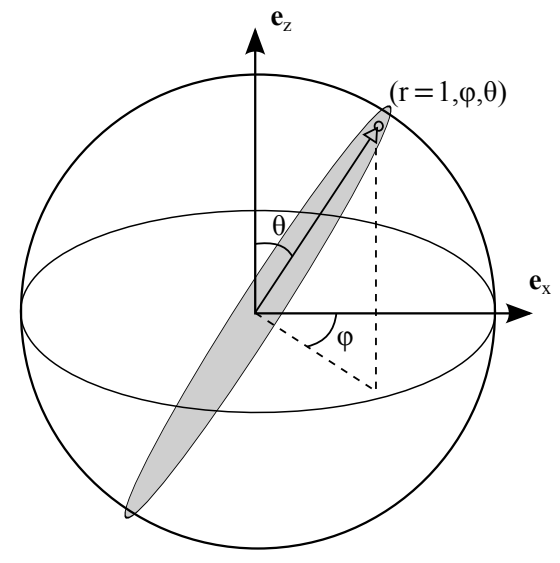

Figure 3: Schematic figure of the unit sphere in which a graphite flake orientation is represented by a unit vector given by the spherical coordinates $(r=1, \varphi, \theta)$. The $\mathbf{e}_{z}$ unit vector is taken as the loading direction.

Thus, the probability density function equals to $1 / 4 \pi$. Furthermore, the expected value of the projected graphite flake length can now be calculated as

$$
\bar{l}_{\perp}=\int_{0}^{2 \pi} \int_{0}^{\pi} l_{\perp} \psi \sin \theta d \theta d \varphi=\frac{\pi}{4} \bar{l}
$$

where $l_{\perp}=\bar{l} \sin \theta$ and $\bar{l}$ is the average flake length. This parameter is calculated from the graphite size distribution obtained from image analysis of microstructural images, see Section 3.1, as

$$
\bar{l}=\sum_{i \in 1-8} x_{i} l_{i}
$$

where $l_{i}$ and $x_{i}$ are the characteristic flake size and the fraction of the total number of graphite particles corresponding to each graphite size group $i$ ranging from 1 to 8 , as defined in the graphite distribution standard ISO 945-1:2008.

Regarding the SGI, the microcracks were only seen to initiate at microshrinkages. Therefore, the initial crack length was taken as the average maximum diameter measured on microshrinkage pores at half-life which were observed to have initiated microcracks.

The final crack length, whose magnitude only has a low impact on the number of cycles to failure [22], were selected as $1 \mathrm{~mm}$ for all investigated 
cast irons, motivated by the observations of Socie and Fash [14]. The estimated values of the initial and final crack lengths are presented in Table 1.

\section{Materials and experimental procedure}

\subsection{Materials and specimens}

A material set of three different types of cast irons were investigated and compared, namely a LGI, CGI and SGI material. The LGI material was an alloyed non-commercial EN-GJL-250 with enhanced mechanical properties at elevated temperatures. The CGI was a fully perlitic EN-GJV-400 and the SGI was a fully ferritic high silicon and molybdenum EN-GJS-SiMo5-1 alloy, both commercially available. The chemical composition of the ENGJV-400 and EN-GJS-SiMo5-1 are given in Table 3, while the composition of EN-GJL-250 is omitted due to commercial confidentiality. The LGI and CGI material were cut from plates with a nominal casting thickness of $16 \mathrm{~mm}$ while the SGI-plates had a nominal casting thickness of $18 \mathrm{~mm}$. Furthermore, the EN-GJS-SiMo5-1 was heat-treated, namely a solution treatment at $900^{\circ} \mathrm{C}$ and a subsequent normalisation, in order to remove potential pearlite content. The specimens were then machined into the desired geometry, see Section 3.2.

The various microstructures with their characteristic graphite shapes of the three materials are displayed in Fig. 4. The images were taken from regions cut out from the parallel section of untested TMF specimens. The shape type and size distributions were determined by the software Axiovision whose results were utilised in Section 2.2.

In addition, samples of the same region were chemically etched using an etching agent based on picric acid and sodium hydroxide in order to visualise the eutectic cells and to measure their characteristic size. The eutectic cell sizes were measured in three images and an average was computed from the three largest cells taken from the three images, see Table 4. The eutectic cell size is an important microstructural parameter which affects the mechanical properties and is therefore worthwhile to present.

Table 3: The measured chemical composition in weight percent of EN-GJV-400 and EN-GJS-SiMo5-1. The iron content is implicit.

\begin{tabular}{ccccccccccc}
\hline & $C$ & $S i$ & $M n$ & $S$ & $P$ & $N i$ & $M o$ & $C u$ & $S n$ & $T i$ \\
\hline EN-GJV-400 & 3.38 & 1.90 & 0.374 & 0.010 & 0.019 & $<0.050$ & $<0.010$ & 0.97 & 0.090 & 0.011 \\
EN-GJS-SiMo5-1 & 3.16 & 4.33 & 0.407 & 0.008 & 0.014 & $<0.050$ & 0.91 & 0.073 & $<0.010$ & 0.017 \\
\hline
\end{tabular}



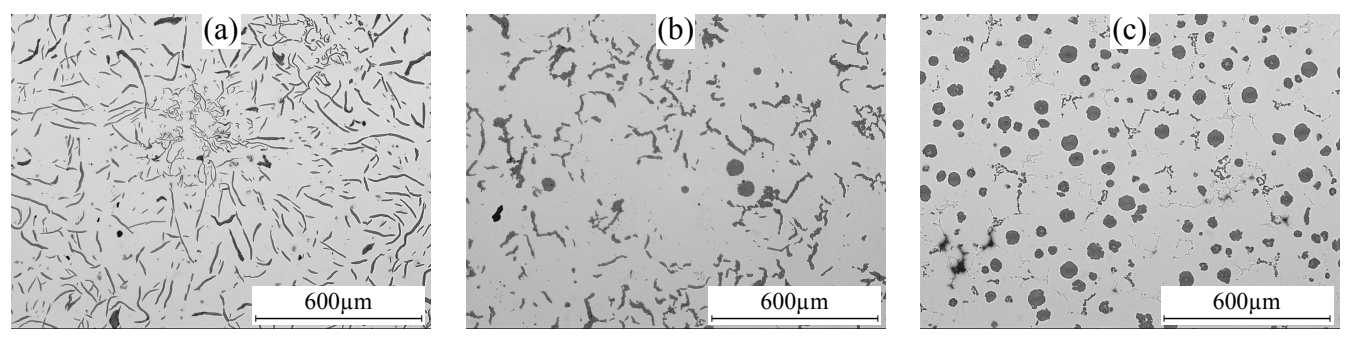

Figure 4: The different microstructures; (a) EN-GJL-250 (b) EN-GJV-400 and (c) ENGJS-SiMo5-1.

Table 4: The measured eutectic cell size represented by the average and standard deviation of the three largest cells found at three different locations.

\begin{tabular}{cc}
\hline Material & Eutectic cell size $[\mu m]$ \\
\hline EN-GJL-250 & $741 \pm 87$ \\
EN-GJV-400 & $293 \pm 17$ \\
EN-GJS-SiMo5-1 & $154 \pm 6$ \\
\hline
\end{tabular}

Typical static mechanical properties of the three materials are presented in Table 2. Regarding the yield and tensile strength, the average and standard deviation data on EN-GJL-250 and EN-GJV-400 are acquired from a large data set of tensile tests done on specimens cut out from components. In the case of EN-GJL-250, $R_{p 0.2}$ is not normally measured in this procedure, and hence not reported here. This is because of the ambiguity when determining the elastic modulus and yield strength of grey irons, which is due to their non-linear elastic behaviour. Conversely, the data presented on EN-GJS-SiMo5-1 is obtained from three tensile tests at room temperature, following the ISO 6892-1:2009 A222 standard, with specimens cut from the cast plate mentioned above. Moreover, the elastic modulus data presented were measured in two tensile tests conducted on each material at $100^{\circ} \mathrm{C}$, according to the ISO 6892-2:2011 A222 standard. The particular test temperature was used since these elastic moduli are required in the presented model, as explained in Section 2. To circumvent the non-linearity problem of EN-GJL-250, the elastic modulus was acquired following the procedure proposed in the ISO 6892-1:2009 standard regarding non-linear elastic materials.

\subsection{Thermo-mechanical fatigue tests}

Thermo-mechanical fatigue tests with and without superimposed highcycle fatigue loading were conducted on all three cast irons using the same 
procedure as described in [10], in accordance with a validated code of practice [3] for TMF testing. The tests consisted of applying repeated thermal and mechanical load cycles in a strain-controlled out-of-phase condition.

The employed TMF cycle consisted of a $200 s$ long ramp up and down, including $25 \mathrm{~s}$ long hold time at each turning point; giving a total cycle period of $450 \mathrm{~s}$. The short cycle period is intentionally selected since tests would otherwise be unreasonably long, and further, the cycle is also motivated by its close resemblance to some of the component tests regularly performed on engine components, such as the cylinder head. In both the TMF and TMF-HCF tests, the minimum temperature $T_{M i n}$ and the maximum total mechanical strain $\varepsilon_{\text {Max }}$ were fixed, namely to $100^{\circ} \mathrm{C}$ and to $0 \%$. The former was chosen due to practical considerations, since it is technically difficult to cool hot specimens to lower temperature in a controlled manner. Regarding the choice of a fixed $\varepsilon_{\text {Max }}$, it is motivated by having a constant $R_{\varepsilon}$-value, defined as $\varepsilon_{\text {Min }} / \varepsilon_{\text {Max }}$, equal to $-\infty$. Moreover, the HCF frequency was chosen as $15 \mathrm{~Hz}$, motivated by the rotational speed of the crank shaft. Similarly, all tested temperature and total mechanical strain loads were selected to be in the vicinity of the conditions found in components in field use or in accelerated testing.

The TMF-HCF tests were varied by keeping the maximum, minimum and range value of the total mechanical strain fixed while varying the $\mathrm{HCF}$ strain range. Consequently, the maximum and minimum TMF strain were free variables allowed to vary freely depending on the choice of $\mathrm{HCF}$ and total mechanical strain variables. Thus all in all, the studied independent variables were the maximum temperature $T_{M a x}$, the total mechanical strain range $\Delta \varepsilon_{M e c h}$ and $\mathrm{HCF}$ strain range $\Delta \varepsilon_{H C F}$.

The TMF and TMF-HCF tests were performed in an Instron 8801 servo hydraulic test machine, in which the materials were heated up by induction heating using an encircling copper coil. The cooling down of the specimens was assisted by a compressed air flow directed towards the specimen through three nozzles. The tests were conducted through automatic computer control using a dedicated TMF software developed by Instron, and the strain was measured using an Instron extensometer.

Smooth cylindrical test specimens were used whose geometry was defined by a $6.3 \mathrm{~mm}$ diameter, $25 \mathrm{~mm}$ parallel length, $12.5 \mathrm{~mm}$ extensometer gauge length and $30 \mathrm{~mm}$ transition radius. The total length of the specimen was $145 \mathrm{~mm}$ and both ends were threaded for gripping with a thread size of M12. The same specimen geometry was used for all three materials. 
The number of cycles to failure $N_{f}$ was defined as the $2 \%$ stress deviation from the tangent drawn at the last point of zero curvature in the stress range curve, as suggested by the validated code-of-practice [3].

\subsection{Metallographic crack investigation}

The evolution of microcracks has been studied on the three considered materials by investigating the microstructure of specimens obtained from interrupted fatigue tests by optical microscopy, namely a Nikon Optiphot optical microscopy at 400 to 1000 times magnification. The specimens were cut along the longitudinal axis, and then again in the cutting plane perpendicular to the first, in order to acquire more cross-sectional surface to study. These surfaces were ground and polished using a standard program for cast irons, which also was the same procedure used in obtaining the microstructure images of Section 3.1. The length of microcracks were measured as the projected length in the direction perpendicular to the loading axis, including the graphite particle from which the crack had initiated. Furthermore, a total area of about $30 \mathrm{~mm}^{2}$ in the centre of each specimen was checked through in the search for microcracks. The chosen TMF condition for the interrupted tests was a $100-500^{\circ} \mathrm{C}$ temperature cycle and a TMF strain range of about $0.35-0.37 \%$. The microcrack length at the end-of-life was measured on completely failed specimens, in which only distinctly isolated microcracks from the fracture surface were considered.

\section{Result and Discussion}

\subsection{Stress-strain behaviour}

Fig. 5 compares the hysteresis loops and the maximum stress for each cycle of the three studied materials subjected to similar load conditions, namely a thermal cycle of $100-500^{\circ} \mathrm{C}$ with a TMF strain range of $0.58 \%$ for EN-GJV-400 and EN-GJS-SiMo5-1 while 0.59\% for EN-GJL-250, and zero HCF strain range. The data of the CGI material has previously been presented in [10]. The difference in attained stress levels due to the prescribed mechanical strain cycle between the materials is clearly observed, with the highest stress values measured in EN-GJS-SiMo5-1 and lowest in EN-GJL-250.

\subsection{Thermo-mechanical fatigue}

Fig. 6a shows a comparison of the TMF life, i.e. the total number of cycles to failure $N_{f}$, as a function of the mechanical strain range for 


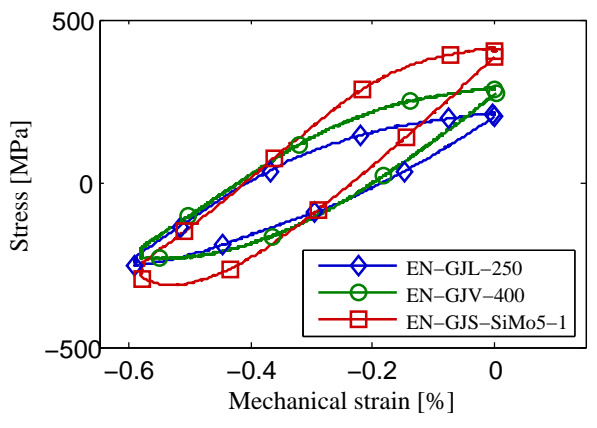

(a)

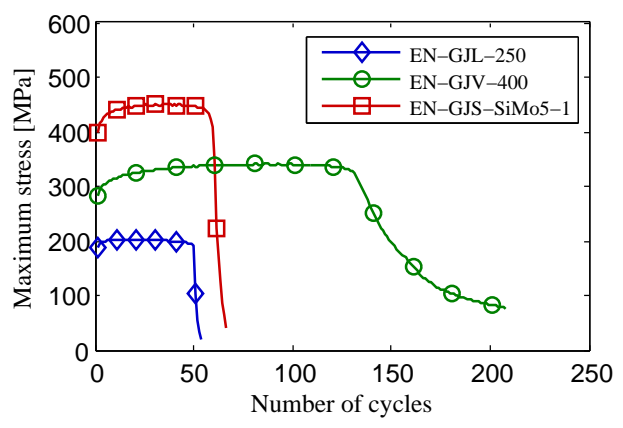

(b)

Figure 5: (a) The hysteresis loop of the second cycle and (b) the maximum stress at each cycle of a TMF test with $100-500^{\circ} C$ thermal cycle, TMF strain range of $0.58 \%$ for EN-GJV-400 and EN-GJS-SiMo5-1 while 0.59\% for EN-GJL-250, and no HCF load, for all three materials.

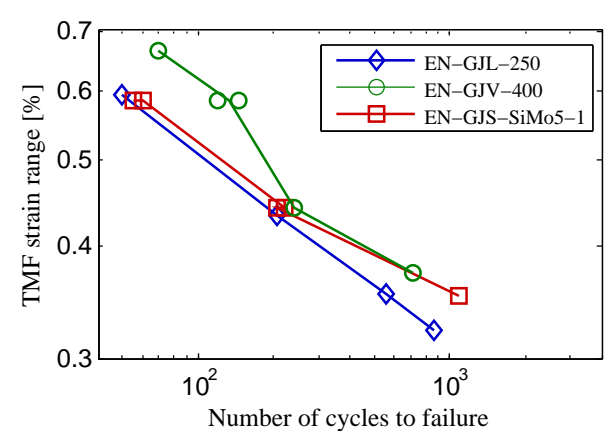

(a)

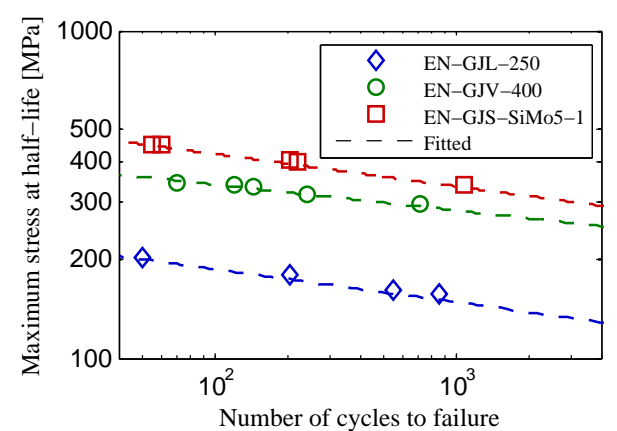

(b)

Figure 6: (a) The strain life and (b) the stress life curves of TMF test with $100-500^{\circ} \mathrm{C}$ thermal cycle and no HCF load, for all three materials.

a thermal cycle of $100-500^{\circ} \mathrm{C}$ and no $\mathrm{HCF}$ loads. The figure compares the TMF life of EN-GJL-250 and EN-GJS-SiMo5-1, as well as data of EN-GJV400 first presented in [10]. No greater difference between the materials is observed, except for a hardly distinguishable improvement going from ENGJL-250 to EN-GJV-400; EN-GJS-SiMo5-1 being somewhat intermediate between the two.

It is important to stress that the material comparison made in Fig. 6a might be misleading, because even if EN-GJS-SiMo5-1 appears to have slightly lower fatigue resistance than the EN-GJV-400 for instance, the former has better overall mechanical properties, see Table 2. Since EN-GJS- 
SiMo5-1 is stronger, i.e. higher elastic modulus and yield strength, and therefore requires higher forces to attain the prescribed deformation, it is very likely that the material will experience lower actual deformation when put into the same operation condition as EN-GJV-400. The reason is that the surrounding is not rigid, but rather compliant with the imposed forces on the considered body-surrounding interface. Thus, the effective deformation and load levels applied on the considered body will be dependent on the combined response of the body-surrounding system. Due to these reasons, Fig. $6 \mathrm{~b}$ is presented considering the stress amplitude response from the experiments instead of the prescribed TMF strain range. This stress amplitude is defined by the cyclic maximum stress measured at half life. Even though it might not be reasonable to assume invariant load conditions between the materials either, a more justified comparison should be to simultaneously considering Figs. $6 \mathrm{a}$ and $6 \mathrm{~b}$.

Looking at Fig. 6b, a much more evident hierarchy is visible, with ENGJS-SiMo5-1 as the most superior material, followed by EN-GJV-400 and then EN-GJL-250. In addition, the number of cycles to failure obtained using Eq. 6, with $\Delta \varepsilon_{H C F}$ equal to zero and the fitted parameters presented in Table 1, are included. This demonstrates the quality of the fit when Eq. 6 is fitted to the TMF tests.

\subsection{Combined thermo-mechanical and high-cycle fatigue}

The TMF-HCF plots seen in Fig. 7 show the reduction in the number of cycles to failure due to the successive increase of the HCF strain range, while keeping the maximum and minimum temperature and total mechanical strain fixed, for EN-GJL-250, EN-GJV-400 and EN-GJS-SiMo5-1. The data of the EN-GJV-400 originates from the previous study [10]. The different curves in Fig. 7 correspond to different values of the maximum temperature and total mechanical strain range, namely $500^{\circ} \mathrm{C}$ or $400^{\circ} \mathrm{C}$ with either $0.44 \%$ or $0.58 \%$ for EN-GJV-400 and EN-GJS-SiMo5-1, or either $0.43 \%$ or $0.59 \%$ in the case of EN-GJL-250. Clearly, all three materials exhibit a TMF-HCF threshold, defined as the HCF strain range where the fatigue life is reduced to half of the value obtained in a TMF test without a $\mathrm{HCF}$ component.

Fig. 7 also contains the estimated number of cycles to failure calculated using the lifetime assessment model presented in Section 2 with the coefficients presented in Table 1. This model estimates the TMF-HCF behaviour well for EN-GJL-250 and EN-GJV-400, Figs. 7a and 7b, however

it underestimates the threshold in EN-GJS-SiMo5-1, Fig. 7c. The reason 


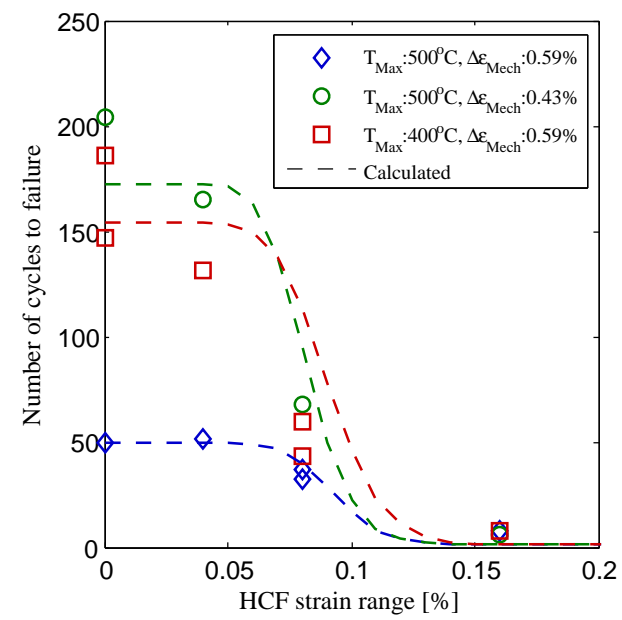

(a)

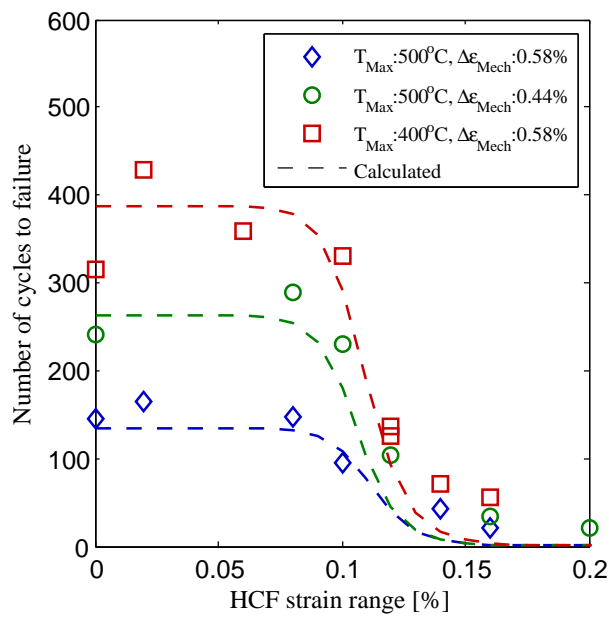

(b)

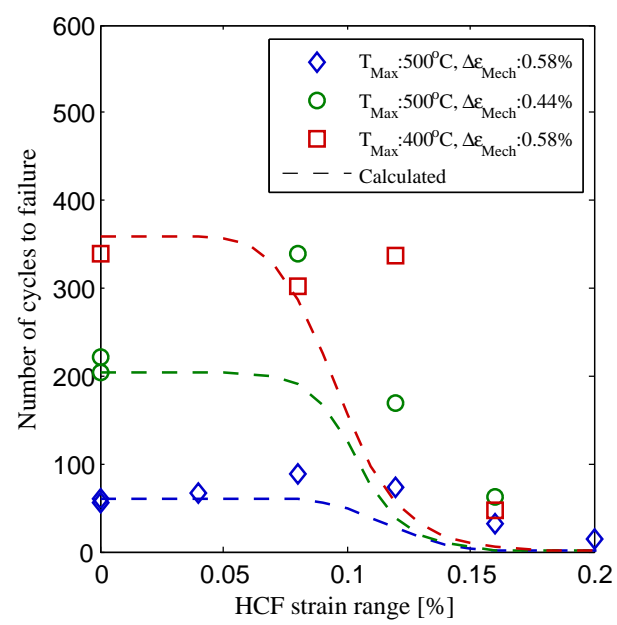

(c)

Figure 7: TMF-HCF plots for (a) EN-GJL-250 (b) En-GJV-400 [10] and (c) EN-GJSSiMo5-1 for different TMF cycles. The dashed lines represent the solutions obtained from Eq. 6 using the corresponding parameters found in Table 1. Note that the y-axis is the number of cycle to failure in contrast to Fig. 6.

might be that the underlying assumptions are oversimplified. For instance, it could be inappropriate to set the Paris parameters of the TMF and the $\mathrm{HCF}$ cycles equal, or to neglect the crack closure effect. More fundamentally, it could be due to the apparent difference in microcrack initiation, 
which is demonstrated below in Section 4.4, that microcracks are initiated at microshrinkage pores rather than at graphite particles. Intuitively, the adjacent residual stress field around a void should be remarkably different compared to the field around a graphite particle during tension-compression loading, since the graphite phase is capable of supporting a compressive load, while a void is not.

It is interesting to see that even though the model is based on Paris law, which presupposes crack propagation even for vanishingly small stressintensity factor ranges, it predicts a step-wise trend for increasing $\mathrm{HCF}$ strain range. The reason for this is the abrupt transition from crack propagation dominated by the TMF cycle to crack propagation dominated by the $\mathrm{HCF}$ cycles, i.e. the first and second term in Eq. 2 respectively. Accordingly, since the $\mathrm{HCF}$ contribution to crack propagation, Eq. 5, increases rapidly with $\Delta \sigma_{H C F}$ due to the exponent $n$, the fatigue life is also rapidly reduced as soon as the TMF and HCF contribution become comparable in size.

As also discussed in [10], it is remarkable that the fitting of the model to the experiments results in such high values of the Paris exponent $n$ which is not normal for metallic materials. For instance, Ghodrat et al. [16] obtained a value of 5 when investigating TMF tests of notched CGI specimen, which they also confirmed to be valid for cracks emanating from notches of the same size as the average graphite size. On the contrary, the specimens tested in the current study were smooth, resulting in the propagation of multiple small cracks, see Section 4.4 below, which could rationalise the high $n$ values. Effectively, small cracks have been shown to behave quite differently from macroscopical cracks, especially due to their microstructure dependence [22].

In Fig. 8 TMF-HCF tests of all three materials are summarised by normalising the curves in Fig. 7 to data points obtained below the TMFHCF threshold. For each material, all curves collapse into a single curve, represented in Fig. 8 as averages. The dispersion is represented by bars representing the maximum deviation from the average. The hierarchy is clearly visible, EN-GJS-SiMo5-1 possessing the highest TMF-HCF threshold, $\Delta \varepsilon_{H C F}^{T h}=0.14 \%$, and EN-GJL-250 the lowest, $\Delta \varepsilon_{H C F}^{T h}=0.08 \%$, while EN-GJV-400 is intermediate with $\Delta \varepsilon_{H C F}^{T h}=0.11 \%$. By reference to Eq. 9, the observed increasing order is argued to be a result of the different cyclic stress levels $\sigma_{\text {Max }}$ manifested by the different cast irons, see Fig. $5 \mathrm{~b}$ in which EN-GJS-SiMo5-1 exhibits the highest stress level. The reason is 
simply that the damage contribution of a $\mathrm{HCF}$ cycle, which scales with $\Delta \sigma_{H C F}$, become less in relation to the contribution of the TMF cycle, i.e. $\sigma_{\text {Max }}$, if the maximum stress was to increase. Conversely, it should be noted that the absolute value of the number of cycles to failure will not necessarily improve even though the threshold is increased with increasing $\sigma_{\text {Max }}$. Since Fig. 8 is normalised, the effect of $\sigma_{M a x}$ on the absolute fatigue life becomes concealed.

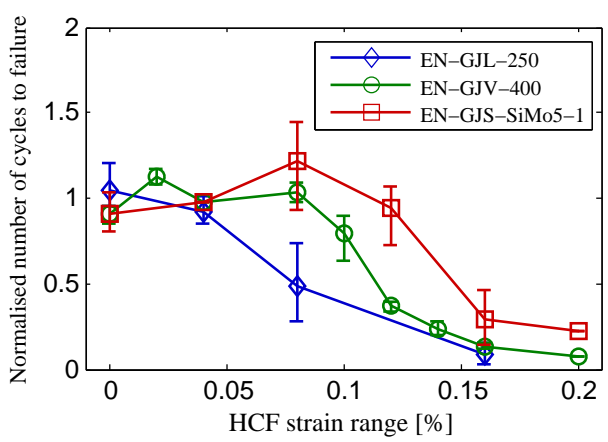

Figure 8: TMF-HCF plot in which the TMF-HCF curves of EN-GJL-250, EN-GJV-400 [10] and EN-GJS-SiMo5-1 in Fig. 7 are normalised with the TMF fatigue lives obtained with $\Delta \varepsilon_{H C F}$ below the TMF-HCF threshold. All curves corresponding to each material are represented by the average and maximum deviation from the average.

An important remark is that the threshold values presented here correspond to a particular $\mathrm{HCF}$ frequency, $15 \mathrm{~Hz}$, since the threshold is very likely to be HCF frequency dependent, as suggested by Eq. 9. This can easily be understood by realising that the HCF frequency will determine the number of HCF cycles per TMF cycle. Hypothetically, the frequency dependence should be possible to be included in Eq. 9, which however remains to be verified.

\subsection{Microcrack growth}

Optical microscope images were taken on specimen obtained from interrupted TMF tests stopped after approximately half of their total fatigue life. Examples of such images are displayed in Fig. 9, where the occurrence of microcracks are visible. By a qualitative comparison, the crack morphology in EN-GJL-250 appears to be similar to EN-GJV-400, as described in [10]; exhibiting microcracks at graphite tips found at multiple locations, both near the surface of the specimen and within the bulk. 

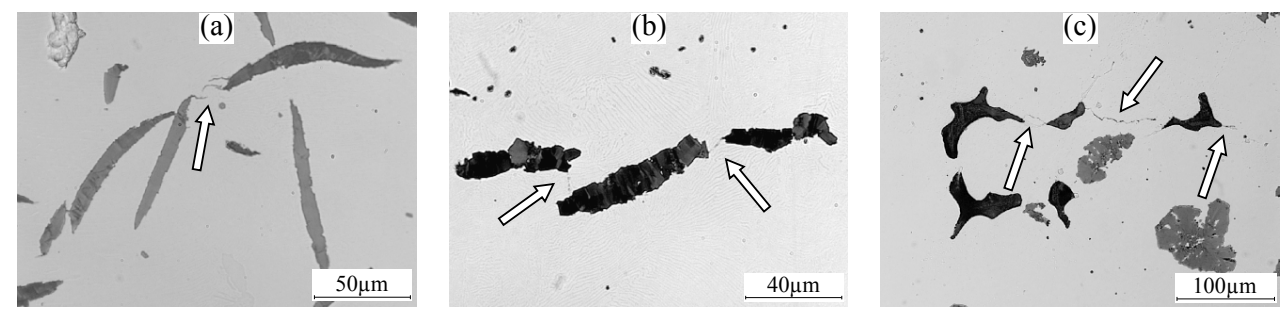

Figure 9: Microcracks observed in (a) EN-GJL-250,(b) EN-GJV-400 and (c) EN-GJSSiMo5-1 after having consumed the half of the total fatigue life of a TMF test with a $100-500^{\circ} \mathrm{C}$ temperature cycle and a TMF strain range of about $0.35-0.37 \%$. The load axis is equal to the vertical axis in these images.

In the EN-GJS-SiMo5-1 however, no microcracks emanating from graphite nodules could be observed. In contrast to EN-GJL-250 and EN-GJV-400, microcracks were encountered at microshrinkage porosities, as seen in Fig. 9. These observations are in accordance with other investigations made on similar SGI materials [17, 18, 23], in which it has been demonstrated that casting defects such as microshrinkage pores often act as fatigue crack initiation sites. No similar microcracks were found at casting defects in ENGJL-250 and EN-GJV-400 apart from a few rare exceptions. Microcracks initiated at defects were therefore not regarded as significant in comparison to the extensive microcrack initiation at the graphite tips in these two materials. Moreover, it is important to emphasise that despite the difference in the initiation site in EN-GJS-SiMo5-1, the number of microcracks was still large due to the relatively high density of microshrinkage pores.

Remarkably, these observations are not in agreement with the findings of Hammers et al. [5] who only reported of cracks emanating from graphite particles or the specimen surface in a similar SGI material, EN-GJS-700, due to TMF and TMF-HCF loading. In addition, comparing Fig. 6a with the strain life curves presented in [5, 7], which was obtained for a similar temperature cycle of $50-450^{\circ} \mathrm{C}$, it is observed that the studied EN-GJS-SiMo5-1 has almost ten times shorter fatigue life than EN-GJS-700. Therefore, it is likely that the reason for the shorter life of EN-GJS-SiMo5-1 is the existence of microshrinkage pores, which seem to be more prone to initiate microcracks compared to graphite particles.

The length of about 15 to 35 microcracks emanating for graphite particles, and microshrinkage pores in the case of EN-GJS-SiMo5-1, was measured using the method described in Section 3.3. Specimens of the three studied materials were obtained from interrupted TMF tests with identical 


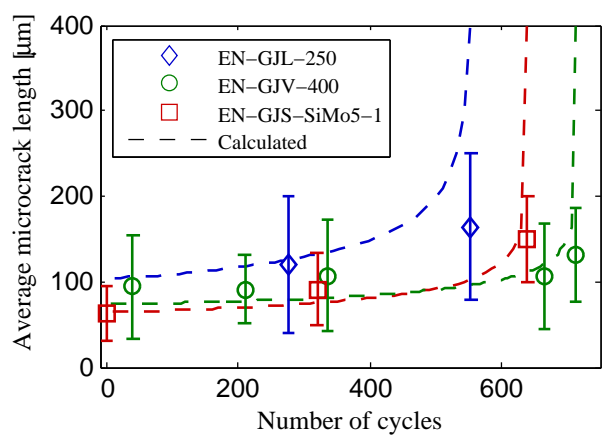

Figure 10: Measurement of the average microcrack length in interrupted TMF tests with a $100-500^{\circ} \mathrm{C}$ temperature cycle and a TMF strain range of about $0.35-0.37 \%$ after different fractions of the fatigue life for EN-GJL-250, EN-GJV-400 and EN-GJS-SiMo51. The markers represent the average and the bars indicate the standard deviation. The dashed lines represent the calculated average microcrack length using Eq. 10.

test conditions, namely a $100-500^{\circ} \mathrm{C}$ temperature cycle and a TMF strain range of about $0.35-0.37 \%$, but stopped after different fractions of the total fatigue life. The evolution of the average microcrack length is presented in Fig. 10 for the three materials, including a measure of variability taken as the standard deviation. Concerning EN-GJV-400, which has the most data points, the average microcrack length only increases mildly with the number of cycles. Similar trends are seen for the average microcrack length in EN-GJL-250 and EN-GJS-SiMo5-1. For all three materials, the average microcrack length measured at end-of-life is within a factor of two compared to the initial average microcrack length, as seen in Fig. 10. Thus, the average microcrack length required for failure is considered as relatively short.

Regarding the considerable scatter, it is due to the fact that microcracks are initiated at various graphite particles and defects, regardless of their size. Thus, the uncertainty seen in Fig. 10 originates from the variability of the size of the crack initiations site rather than the microcrack length. It should also be mentioned that the average length in EN-GJS-SiMo5-1 at the endof-life was significantly affected by a single long microcrack extending up to almost one millimetre. However, this particular crack was still isolated from the main fracture surface, and was thus not responsible for the final fatigue failure.

The presented attempt in characterising the length of microcracks should be considered with caution. It is important to remark that the measurement 


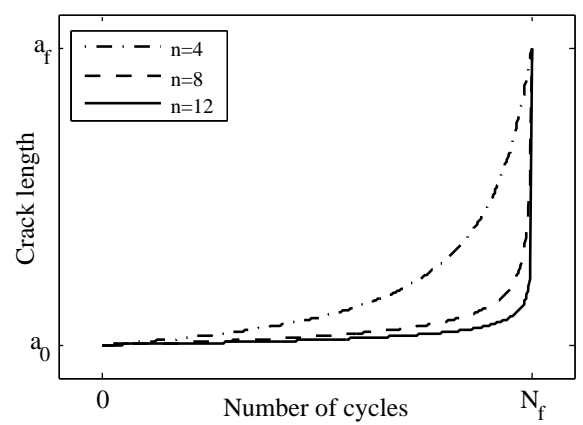

Figure 11: Plot with linear axes of crack length profiles obtained by solving Paris law for different values of the Paris exponent $n$, provided that the stress amplitude is constant and that the number of cycles to failure, initial and finial crack length are known.

is made on two-dimensional surfaces where the three-dimensional nature of the microcracks is left unattended. In addition, it was not always easy to distinguish microcracks from other microstructural features. Regarding the shortest microcracks in particular, they had a significant impact depending on if they were included in the average value or not. Therefore, only the most distinct microcracks were considered, thereby neglecting a large number of features looking like cracks.

The average crack length as a function of number of cycles generated by the lifetime assessment model, as explained in Section 2.2, using the parameters, the initial and final crack length in Table 1 for $100-500^{\circ} \mathrm{C}$, is also included in Fig. 10. The predicted average crack size seems justified by the experimentally measured crack lengths, especially regarding the shape of the curve profile. On the contrary, it might be inappropriate to persevere with the view of microcrack plurality in the case of EN-GJS-SiMo5-1, since one could argue that it is simply the largest defect which determines the life. On the other hand, the experimental study performed has indicated that a large number of microcracks are initiated, approximately 25 defectinitiated microcracks over a cross-sectional surface of $75 \mathrm{~mm}^{2}$ after half the fatigue life, which suggests that also the defect density has significance for the fatigue life.

Coming back to the discussion of the high $n$-value, it is noted that when the Paris relation, Eq. 3, is integrated, the crack length profile $a(N)$ gets a more distinct "knee" for higher $n$, see Fig. 11. Consequently, the crack propagation rate $d a / d N$ becomes lower for small $N$, however also accelerates faster as $N$ passes the knee. Thus, the high $n$-value can be 


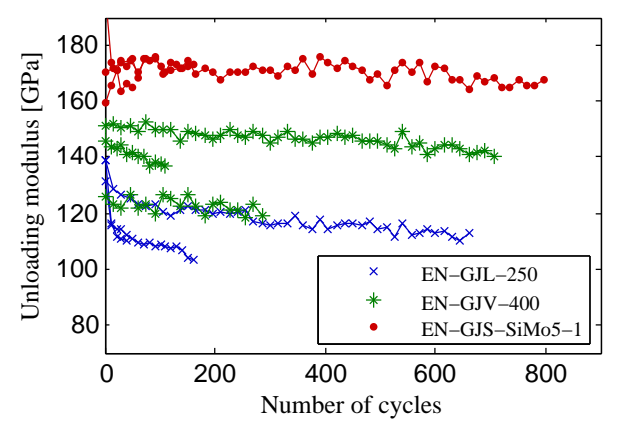

(a)

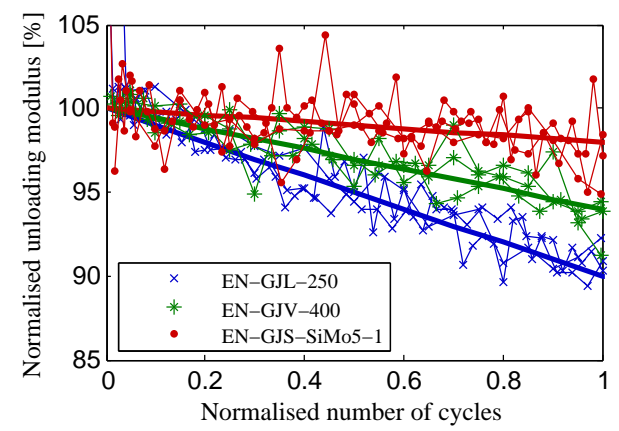

(b)

Figure 12: (a) The unloading modulus of tests conducted in LCF (only in the case of ENGJV-400), TMF and TMF-HCF conditions with different load parameters, as a function of number of cycles, for EN-GJL-250, EN-GJV-400 [15] and EN-GJS-SiMo5-1, and (b) the normalised curves with reference to the initial unloading modulus and the number of cycles to failure. In the latter, a trend-line of each material is included.

motivated by the presented fatigue crack growth behaviour in cast irons, i.e. the extended small fatigue crack growth regime followed by a rapid regime of crack coalescence near the end-of-life. It is also interesting to see that the knee in the EN-GJS-SiMo5-1 curve seen in Fig. 10, coincides with the measured small crack transition size of $120 \mu m$ identified by Nadot [17], corresponding to the microcrack size where the propagation rate becomes comparable to macroscopic cracks. Thus, it is again indicated that the fatigue life of EN-GJS-SiMo5-1 is consumed in the small crack regime.

\subsection{Unloading modulus}

In the paper by Norman et al. [15], a correlation between the amount of microscopic crack growth and the instantaneous value of the elastic modulus measured during unloading was established, as first anticipated by Haenny and Zambelli [24]. One of the important remarks was the consistency in which EN-GJV-400 manifested the same percentage loss in the unloading modulus when the end-of-life was reached, namely a linear decrease to about 5-7\% at the end-of-life. It was argued that the linear regime corresponds to the propagation of multiple microcracks supposedly independent of each other, whilst the depart of the linear regime constitutes the onset of microcrack coalescence and the formation of a dominant macroscopic crack, eventually being responsible for the final failure.

In the present study, this mechanical behaviour has been investigated further by including EN-GJL-250 and EN-GJS-SiMo5-1. The unloading 
modulus versus the number of cycles can be seen in Fig. 12a and the normalised version in Fig. 12b, where the $\mathrm{x}$ and $\mathrm{y}$ axis are normalised to the number of cycles to failure and the unloading modulus measured during the first cycle respectively. The curves included are different tests conducted under different conditions, namely TMF and TMF-HCF conditions with different maximum temperatures and total mechanical strain ranges, and even one LCF test is included in the case of EN-GJV-400. Despite the large variety of test conditions, all materials exhibit a linear decrease, which when normalised collapses into one unique curve for each material, see Fig. $12 \mathrm{~b}$. However, the gradients of these three curves are different; EN-GJL-250 has lost about 9-11\%, EN-GJV-400 about 5-7\% and EN-GJS-SiMo5-1 about $2-4 \%$ of the initial unloading modulus when failure occurs.

In line with the previous argumentation [15], this behaviour is believed to be rationalised by the nature of the microcrack initiation and propagation phenomena. As microcracks become longer, the load carrying area is reduced which leads to a loss in the stiffness of the specimen. Accordingly, the most likely explanation for the apparent difference in the unloading modulus gradient is a possible difference in microcrack density among the cast iron grades; a larger density implying a larger loss in load carrying area as microcracks propagate independently of each others. Accordingly, EN-GJS-SiMo5-1 has the lowest slope most likely because the microshrinkage density in EN-GJS-SiMo5-1 is much lower than the graphite particle density in EN-GJL-250 and EN-GJV-400.

The results presented in Fig. $12 \mathrm{~b}$ is a clear indication that the fatigue processes under TMF, TMF-HCF and possibly also LCF conditions, follow a general pattern in cast irons. In other words, it is reasonable to believe that microcracks initiate and propagate in a similar manner regardless of the specific load condition. However, it is further argued that the parameters associated with any of these load conditions, e.g. the total mechanical strain range and the temperature cycle, still affect the rate of the general fatigue process.

\section{Conclusions}

- The effect of a superimposed HCF strain load on a strain-controlled out-of-phase TMF test configuration has been studied on three different cast irons, namely a lamellar graphite iron, EN-GJL-250, a compacted graphite iron, EN-GJV-400 and a spheroidal graphite iron, ENGJS-SiMo5-1. The relatively unstudied TMF-HCF property, namely 
the TMF-HCF threshold $\Delta \varepsilon_{H C F}^{T h}$, defined as the HCF strain range at which the fatigue life is reduced to half the value of the corresponding TMF test without superimposed $\mathrm{HCF}$, has been identified and measured to $0.08 \%, 0.11 \%$ and $0.14 \%$ for EN-GJL-250, EN-GJV-400 and EN-GJS-SiMo5-1 respectively, with a $\mathrm{HCF}$ frequency of $15 \mathrm{~Hz}$. Thus, it is concluded that EN-GJS-SiMo5-1 is superior in resisting TMF-HCF load conditions.

- The fatigue cracking process associated with TMF loading has been studied and it has been observed that the damage accumulation is characterised by multiple microcrack initiation and propagation in all the studied materials. In contrast to the other two, microcracks are initiated at microshrinkage pores in EN-GJS-SiMo5-1 instead of the graphite-matrix phase boundary, however still at multiple locations, since pores are densely and quite homogeneously distributed.

- A TMF-HCF lifetime assessment model based on Paris law and developed in a previous paper [10], was used successfully to estimate the fatigue life of laboratory specimen subjected to both TMF and TMFHCF load conditions. However, while giving good life estimates for EN-GJL-250 and EN-GJV-400, the model underestimates the TMFHCF threshold in EN-GJS-SiMo51. Nevertheless, the model was justified by comparison to metallographic measurements of the average microcrack length in TMF conditions, and from the model, an expression of the TMF-HCF threshold was derived which supposedly can estimate the HCF frequency dependence and the TMF-HCF threshold in other cast irons.

\section{Acknowledgement}

The present study was financed by Scania CV AB, the Swedish Governmental Agency for Innovation Systems (FFI - 2012 - 03625), and the Swedish Foundation for Strategic Research $(S M 12-0014)$. Agora Materiae and the Strategic Faculty Grant AFM (SFO - MAT - LiU\#2009-00971) at Linköping University are also acknowledged. Special thanks are also addressed to Dr. Jessica Elfsberg for assisting in the chemical etching work, Patrik Härnman for his technical support on the TMF machine, and the project group at Scania for all their comments and feedback. 


\section{References}

[1] S. Trampert, T. Gocmez, S. Pischinger, Thermomechanical Fatigue Life Prediction of Cylinder Heads in Combustion Engines, Journal of Engineering for Gas Turbines and Power 130 (012806) (2008) 1-10.

[2] H. Sehitoglu, Thermo-mechanical fatigue life prediction methods, in: ASTM Special Technical Publication, 1992, pp. 47-76.

[3] P. Hähner, E. Affeldt, T. Beck, H. Klingelhöffer, M. Loveday, C. Rinaldi, Research and development into a European code-of-practice for strain-controlled thermomechanical fatigue testing, International Journal of Fatigue 30 (2) (2008) 372-381.

[4] J. J. Thomas, L. Verger, A. Bignonnet, E. Charkaluk, Thermomechanical design in the automotive industry, Fatigue and Fracture of Engineering Materials and Structures 27 (10) (2004) 887-895.

[5] T. Hammers, A. Uihlein, K. Lang, D. Löhe, Development of damage in cast iron during superimposed low frequency thermal-mechanical and higher frequency mechanical loading, in: Plasticity, Failure and Fatigue in Structural Materials-from Macro to Nano: Hael Mughrabi Honorary Symposium, New Orleans, 2008, pp. $253-258$.

[6] A. Uihlein, K. Lang, D. Löhe, Lifetime Behavior at Superimposed ThermalMechanical and Mechanical Loading, in: Proceedings of the XIth International Congress and Exposition, 2008, pp. 306-313.

[7] T. Beck, K. Lang, D. Löhe, Interaction of thermally induced and mechanical fatigue, Transactions of The Indian Institute of Metals 63 (2-3) (2010) 195-202.

[8] M. Metzger, B. Nieweg, C. Schweizer, T. Seifert, Lifetime prediction of cast iron materials under combined thermomechanical fatigue and high cycle fatigue loading using a mechanism-based model, International Journal of Fatigue 53 (2013) 58-66.

[9] C. Schweizer, T. Seifert, B. Nieweg, P. von Hartrott, H. Riedel, Mechanisms and modelling of fatigue crack growth under combined low and high cycle fatigue loading, International Journal of Fatigue 33 (2) (2011) 194-202.

[10] V. Norman, P. Skoglund, D. Leidermark, J. Moverare, Thermo-mechanical and superimposed high-cycle fatigue interactions in compacted graphite iron, International Journal of Fatigue 80 (2015) 381-390.

[11] M. Metzger, M. Leidenfrost, E. Werner, M. Riedler, T. Seifert, H. Riedel, Lifetime Prediction of EN-GJV 450 Cast Iron Cyder Heads under Combined ThermoMechanical and High Cycle Fatigue Loading, SAE International Journal of Engines 7 (2) (2014) 1073-1083.

[12] R. Voigt, C. jun. Loper, Microstructural aspects of fracture in cast irons, in: Physical Metallurgy of Cast Iron IV, Proceedings 4th International Symposium, Tokyo, Japan, 1989, pp. 293-303.

[13] Z. He, S. Ji, G. Lin, Mechanical behaviour of graphite and its adjacent zone to matrix interface in cast iron, Acta metallurgia sinica 6 (3) (1993) 364-368.

[14] D. Socie, J. Fash, Fatigue behavior and crack development in cast iron, Tech. rep., Department of Mechanical and Industial Engineering, University of Illinois at Urbana-Champaign, Urbana (1982).

[15] V. Norman, P. Skoglund, J. Moverare, Damage Evolution in Compacted Graphite Iron during Thermo- Mechanical Fatigue Testing, International Journal of cast metals research 00 (0) (2015) 00-00, in press. doi:10.1179/1743133615Y.0000000019. 
[16] S. Ghodrat, A. Riemslag, M. Janssen, J. Sietsma, L. Kestens, Measurement and characterization of Thermo-Mechanical Fatigue in Compacted Graphite Iron, International Journal of Fatigue 48 (2013) 319-329.

[17] Y. Nadot, N. Mendez, N. Ranganathan, A. Beranger, Fatigue life assessment of nodular cast iron containing casting defects, Fatigue and Fracture of Engineering Materials and Structures 22 (4) (1999) 289-300.

[18] Y. Nadot, J. Mendez, N. Ranganathan, Influence of casting defects on the fatigue limit of nodular cast iron, International Journal of Fatigue 26 (2004) 311-319.

[19] S. Ghodrat, M. Janssen, R. H. Petrov, L. A. I. Kestens, Microstructural Evolution of Compacted Graphite Iron under Thermo-Mechanical Fatigue Conditions, Advanced Materials Research 409 (2012) 757-762.

[20] H. Kitagawa, S. Takahashi, Applicability of fracture mechanics to very small cracks or the cracks in the early stage, in: Second International Conference on Mechanical Behavior of Materials, ASM, Ohio, 1976, pp. 627-631.

[21] P. Clement, J. P. Angeli, A. Pineau, Short Crack Behaviour in Nodular Cast Iron, Fatigue \& Fracture of Engineering Materials and Structures 7 (4) (1984) 251-265.

[22] S. Suresh, Fatigue of materials, 2nd Edition, Cambridge university press, Cambridge, 1998.

[23] M. Endo, K. Yanase, Effects of small defects, matrix structures and loading conditions on the fatigue strength of ductile cast irons, Theoretical and Applied Fracture Mechanics 69 (2014) 34-43.

[24] L. Haenny, G. Zambelli, Strain mechanisms in grey cast iron, Engineering Fracture Mechanics 18 (2) (1983) 377-387. 